\title{
Helen Salisbury: Risk taking and decision making
}

\author{
Helen Salisbury GP \\ Oxford
}

My patient was coughing but is better now. Meanwhile, blood tests reveal that, although there are no signs of infection, she's mildly anaemic. Should I repeat the test? Add some other blood tests to look for a cause? Check for blood in the stool? What I decide to do, and how soon, will be a matter of judgment, taking into account exact numbers, deviation from previous results, her other health problems, and what she herself thinks. We may also consider how easy it is for her to come to the surgery for further tests.

These are the sorts of decisions GPs take dozens of times every day while rapidly, almost unconsciously, weighing up the risks and costs to the patient and the service. In 2003 David Haslam described general practice as the "risk sink" of the NHS ${ }^{1}$-an analogy to the heat sink of a computer. This component may not seem to be doing much, but without it the whole machine overheats and ceases to function.

Medical practice can't be $100 \%$ safe: almost all of our activity carries risk, from bruising after a blood test to a serious adverse reaction caused by a drug. If we investigated every symptom that our patients present with for every possible cause, hospital laboratories would be overwhelmed and waiting times for radiology would be even worse than now-this clogged-up system becoming a danger in itself.

One of a GP's main jobs, now and always, is to prioritise. When should we investigate without delay to exclude a rare but serious illness? When is it safe to watch and wait? This may vary with the patient we're seeing: the archetypal farmer with "a spot of bother" may be more seriously ill than the understatement implies. Deciding which, if any, of the eight feverish children I see on a duty day need hospital attention, which ones I need to keep an eye on, and which will almost certainly be OK is a skill that takes training and practice. No algorithm can do this yet, and protocol driven triage such as NHS 111 inevitably sends more patients to the emergency department than need to be there, because it has to be safe.

If general practice is to continue as the "risk sink" of the NHS we need staff to be adequately trained, supported, and indemnified. The decisions we take-what symptoms to investigate, how to react to abnormal results, when to start and stop treatments-are complex and require an understanding of physiology and pharmacology, not to mention psychology, which takes time to acquire. I'm concerned that recent proposals for other healthcare professionals to have a shortened medical training to qualify as doctors may lead to them lacking the knowledge, skills, and confidence needed for this work. ${ }^{2}$

\section{Competing interests: See www.bmj.com/about-bmj/freelance-contributors.} Provenance and peer review: Commissioned; not externally peer reviewed.

Haslam D. "Schools and hospitals" for "education and health". BMJ 2003;326:234-5. 10.1136/bmj.326.7383.234 12560251

2 Philpotts E. DH plans for pharmacists and paramedics to retrain as doctors in three years. Pulse 2020 Jan 31. http://www.pulsetoday.co.uk/news/all-news/dh-plans-for-pharmacistsand-paramedics-to-retrain-as-doctors-in-three-years/20040075.article. (Login needed.)

Published by the BMJ Publishing Group Limited. For permission to use (where not already granted under a licence) please go to http://group.bmj.com/group/rights-licensing/ permissions 